Bull. Mater. Sci., Vol. 4, Number 3, May 1982, pp. 267-282. (C) Printed in India.

\title{
Diffusion kinetics and some aspects of solidification under microgravity environment
}

\author{
P RAMACHANDRARAO \\ School of Materials Science and Technology, Banaras Hindu University, \\ Varanasi 221005 , India \\ MS received 21 April 1980
}

\begin{abstract}
The paper surveys the available literature on the direct influence of microgravity on diffusion in liquid metals, rate of solidification, growth of dendrites, undercooling of liquid metals and alloys and monotectic solidification. Agreement between theoretical predictions and experimental observations is discussed critically and areas requiring further study are highlighted.
\end{abstract}

Keywords. Diffusion kineties; solidification rate; dendrites growth; monotectic solidification.

\section{Intradaction}

Materials processing in space has several distinct technological advantages such as containerless handling, reduced convection arising from compositionally and thermally induced density gradients, lack of sedimentation and absence of hydro* static pressure in addition to the availability of high vacuum. All these advantages can be exploited fully in solidification processing. Many topics such as crystal growth, eutectic solidification and composite growih are dealt with at length in other articles in this volume. The present paper is confined to the role of microgravity in controlling diffusion, growth of dendrites, solidification of under. cooled melts and monotectic freezing. Available literature is surveyed and possible experiments are indicated.

\section{Diffusion}

Under terrestrial conditions, diffusion in liquid metals and alloys is influenced by the existence of convective flow. Under microgravity conditions and in the absence of convective flow, liquid diffusion becomes the most important factor in controlling solidification. In this context, an estimation of the direct influence of acceleration forces on liquid state diffusion coefficient assumes importance.

\subsection{Liquid state diffusion}

Miller and Ruff (1975) have employed the free volume model of liquids due to Cohen and Turnbull (1959) for theoretioally predicting the influence of micro- 
gravity on the self-diffusion coefficient in liquid metals. The model assumes that atomic transport in a hard sphere fluid becomes possible only when a void of volume greater than a critical value $v^{*}$ is formed. The critical void is postulated to arise from a redistribution of the total free volume $N v_{f}$ where $v$, is defined by

$$
v_{i}=v-v_{0}
$$

and $N$ is the Avagadro number, $v$ is the volume per atom in the liquid and $v_{0}$ is the atomic volume calculated from atomic diameter. The redistribution of free volume is considered to take place without any attendant changes in energy at constant volume. Assuming a random partitioning of the free volume, Cohen and Turnbull (1959) obtained the following expression for the diffusion coefficient $D$ :

$$
D=\phi T^{1 / 2} \exp \left[-\left(\frac{\mu v^{*}}{v}\right)\left(1-\frac{v_{0}}{v}\right)^{-1}\right],
$$

where $\phi$ is a material constant, $T$ is the absolu te temperature and $\gamma$ is an overlap factor with a. value between $\frac{1}{2}$ and 1 .

Miller and Ruff (1975) first obtsined the ratio of the diffusion constant under microgravity $\left(D^{\prime}\right)$ to that under terrestrial conditions $(D)$ on the basis of $(2)$. The resultant expression is

$$
D^{\prime} \mid D=\left(1+\frac{\Delta T}{T}\right)^{1 / 2} \exp \left\{\frac{\gamma v^{*}}{v}\left[\frac{\frac{\Delta v}{v}}{\left(1-\frac{v_{0}}{v}\right)^{2}+\left(\frac{\Delta v}{v}\right)\left(1-\frac{v_{0}}{v}\right)}\right]\right\}
$$

where $\triangle T$ and $\Delta v$ are changes in $T$ and $v$ due to external field effects. It was further argued that the shifting of a container of liquid from earth to an orbiting laboratory will result in a change in the hydrostatic pressure of

$$
\triangle P \approx-\rho g Z
$$

where $Z$ is the reference pressure point below the free surface of liquid on earth, $\rho$ is the liquid density and $g$ is the acceleration due to gravity. Together with the definitions of isothermal compressibility $(\beta)$ and thermal expansion, coefficient $(a)$ we have:

$$
\triangle v / v=\beta \rho g Z
$$

and $\quad \triangle T T=\frac{(\triangle v / v)}{a T}$.

A knowledge of $(\Delta v / v)$ and $(\Delta T / T)$ is sufficient to estimate the fractional change in the diffusion coefficient due to changes in gravitational field. Estimation of the ratio $D^{\prime} \mid D$ for $\mathrm{Ge}, \mathrm{Hg}$ and $\mathrm{Na}$ indicates that the increase in $D^{\prime}$ is in the range of $10^{-5} \%$. Thus, Miller and Ruff (1975) conclude that reducing the gravitational field has an insignificant effect on the diffusion coefficient in the liquid state. Any changes in $D$ can only be attributed to the absence of convection.

A study of the radioactive isotope tracer diffusion in molten zine (Ukanwa, 1974) indicated no measurable convection effects under microgravity conditions. In another experiment carried out by Reed et al (1977) on Appollo-Soyuz Test Project (ASTP) diffusion of gold in lcad was studied. A liquid diffusion 
couple was formed by a cylinder of lead and a thin disc of lead containing 0.05 at \% Au. On irradiation, Au 198 isotope was obtained and used to study diffusion behaviour by autoradiography. In samples produced in the space flight, a sharp interface of gold in lead could be detected at a distance of $1 \cdot 2 \mathrm{~cm}$. In ground-based experiments, gold was found to be confined to the bottom of the cylinder only. It was shown that Fick's laws of diffusion are sufficient to explain the observed concentration profiles. The analysis was, however, criticised by Carruthers (1977) who noted that the boundary conditions as well as the use of zinc self-diffusion data in the analysis of compositional profiles are questionable.

While the above experiments indicate the absence of residual convection, studics on solute microsegregation in gallium doped single crystals of germanium grown in space prove that some solute mixing does occur under conditions of microgravity (Yue and Voltmer 1975). While the crystals grown in space had a six-fold improvement in macrosegregation and nearly five-fold betterment in microsegregation, the very presence of microsegregation was taken to imply the existence of solute mixing. This non-gravity-driven mixing can arise from Marangoni and Soret effects, temperature induced microdensity thuctuations and phase change convections.

Longitudinal and lateral macrosegregation variations observed in ASTP experiments have also brought to light a new macrosegregation phenomenon. Under conditions of little or no convection, segregation is considered to arise from a lateral component of diffusion at a curved growth interface. Variation in heat flow can result in changes in interface curvature and produce changes in lateral and longitudinal segregation of this type (Carruthers 1977).

\subsection{Sedimentation and diffusion}

In a binary melt in which an interplay of gravity-dependent concentration driven convection and sedimentation exists, it is difficult to estimate (from diffusion theory) the time required for complete homogenisation of the liquid after a compositional fluctuation (Ang and Lacy 1979). For example, in the case of a sinusoidal fluctuation in the concentration $C$ of one of the components with distance $x$, a solution of Fick's second law is

$$
C / C_{m}=\sin \left(\frac{\pi x}{L}\right) \exp \left(\frac{\pi^{2} D t}{L^{2}}\right),
$$

where $C$ is the maximum initial variation in $C, L$ is the effective diffusion distance between the two phases, $D$ is the temperature-dependent diffusion coefficient and $t$ is the homogenisation time (Carruthers, 1977). Application of the equation to Al-Sb melts (Ang and Lacy 1979), which represent the class of liquids under consideration, shows that even when the compositional fluctuation yields a pure phase, a soaking period of 15 min should homogenise the melt at temperatures where $D=1 \times 10^{-5} \mathrm{~cm}^{2} \mathrm{sec}^{-1}$. In practice, however, even holding for as long as $4 \mathrm{hr}$ is insufficient to bring about uniformity in composition.

Ang and Lacy (1979) have developed an elegant analysis in which a parameter $H$ defined by

$$
H=\frac{\text { sedimentation distance }}{\text { diffusion distance }}=\frac{d^{2} \Delta \rho g}{6 \pi v}\left[\frac{t_{s}}{D}\right]^{1 / 2}
$$


takes into consideration the competitive mechanisms of sedimentation and diffusion. With respect to the $\mathrm{Al}-\mathrm{Sb}$ melt, $d$ represents the average diameter of the $\mathrm{Al}-$ and $\mathrm{Sb}$-rich phases, $\Delta \rho$ is the density difference between these phases and Al-Sb melt of viscosity $v$ and $t_{s}$ is the soaking time. A reduction, by about four orders of magnitude in $g$, makes $H \ll 1$ in space flight experiments and should, therefore, enable homogenization in a time predicted by (7) while $H \gg 1$ in groundbased experiments hinders restoration of uniformity in composition and leads to a failure of (7).

Experiments performed by Ang and Lacy (1979) on the ASTP mission showed that there is a five-fold improvement in the homogeneity of Al-Sb specimen solidified in space. The samples obtained in ground-based experiments contained significant quantities of Al- and $\mathrm{Sb}$ - rich phases. These form at the grain boundaries due to segregation on a macroscopic scale. The tracc accurrence of such phases in the low gravity samples attributed to a loss of stoichiometry due to evaporation of antimony. Appropriate compensation prior to solidification could result in the production of chemically and microstructurally homogeneous Al-Sb. The results are of considerable technological importance in view of the high $\left(25^{\circ} \mathrm{f}\right)$ efficiency of $\mathrm{Al}-\mathrm{Sb}$ when used as a solar cell material.

\subsection{Diffusion in two-phase solid materials}

Solutes in a two-phase solid can be redistributed by the existence of electro- and thermomigration and the presence of gravitational fields. Under such conditions, volume diffusion is predominant and the second phase dissolves, maintains saturation levels of solute and reprecipitates elsewhere. This results in a velocity $\psi$ for the precipitate particles. The evolution of second phase in an infinite sample subjected to gravitational fields has been analysed by Stark (1978) who showed that the space and time dependence of the volume fraction of a second phase, $\phi(x, t)$ exhibits a wave-like behaviour according to

$$
\phi(x, t)=\phi_{0}\left[x+\left(\frac{J^{\prime}}{\rho}-\psi\right) t\right]
$$

where $\phi_{0}=\phi(x)$, the initial fraction, $x$ is the distance, $J^{\prime}$ is the steady state flux and $\rho$ is the number density of solute in the second phase. No experiments either at low ar high gravity levels appear to have been performed so far to test the model.

\section{Solidification}

Solidifioation is an essentially nucleation and growth phenomenon and an estimation of the role of microgravity on these two stages would be of significance. Malmejac (1978) has catalogued the thermodynamic parameters and driving forces that control nucleation and attempted to analyse the anticipated effects of microgravity on the role of foreign particles as well as convection and compositional fluctuations in influencing nucleation in liquids. Thermadynamic and energetic factors such as critical embryo size and shape, bulk and surface free energies, degree of undercooling and volume of the material are expected to be unaffected by the decrease in gravitational forces. Increase in solute supersaturation of the 
liquid phase, vapour pressure over the system and solute distribution coefficient can enhance nucleation and are also prone to be altered in the absence of gravity. Lack of convection can result in a thicker boundary layer at the solid-liquid interface and could affect the growth morphology.

The analysis of Miller and Ruff (1975) on the influence of microgravity on the rate of growth of a rough interface indicates that no significant changes should occur. They arrive at the conclusion on the basis of Turnbull's (1961) model according to which the rate of growth is given by:

$$
U=D\left(f_{0} / R_{0}\right)[L-\exp (\triangle G / k T)],
$$

where $f_{0}$ is the fraction of lattice sites on the interface where attachment af atoms/ molecules can accur and $R_{0}$ is the molecular diameter. $\triangle G$ is the Gibb's free energy of solidification at temperature $T$ and $k$ is the Boltzmann's constant. Once again, estimation of $D, f_{0}$ and $\triangle G$ under microgravity conditions will facilitate an assessment of the relative changes in growth rate due to changes in gravitational forces. It was shown that

$$
\begin{gathered}
f_{0} / f_{0}^{\prime}=\frac{\triangle G^{\prime} \mid \triangle G}{1+\frac{\triangle v}{v}},
\end{gathered}
$$

and $\triangle G^{\prime}=\triangle G-\beta v(\triangle P)^{2}$,

where the primes denote the parameters under microgravity conditions while the other parameters have the same meaning as in (5) and (6). The analysis enabled Miller and Ruff (1975) to conclude that the solidification rate will also be affected to an insignificant extent as in the case of the diffusion coefficient. Thus, microgravity is not expected to have any direct influence on the solidification rate. All anticipated and observed changes should arise due to other factors such as reduction in convection, changes in distribution coefficients, etc. For example, unusual growth rate fluctuations in the production of germanium single crystals in ASTP and Skylab flights have been attributed to initial segregation transients associated with heat flow variations (Carruthers 1977; Yue and Voltmer 1975).

Johnston and Baldwin (1974) have chosen the procedure of enhancing the gravitational acceleration to an extent where its effects on nucleation and growth can be magnified to discernible levels. In their experiments, tin crystals were grown at acceleration levels of $1-7 \mathrm{~g}$ produced by centrifuging. It was cbserved that the $c$-axis of the tin crystals is parallel to the acceleration gradient at levels in the range $1 \sim 4 \mathrm{~g}$. This is not the preferred orientation at $1 \mathrm{~g}$ and required an explanation. A consideration of the buayancy and weight forces acting on a nucleus suspended in liquid tin shows that a torque (dependent on the relative orientation of the nucleus with respect to acceleration axis, the density differences between liquid and solid and the prevalent acceleration gradient) arises and aligns the long axis of the growing nucleus along the acceleration axis. Such an alignment is lost at acceleration levels $>4 \mathrm{~g}$ due to acceleration induced convection. Other significant observations of $J_{O} h n s t o n$ and Baldwin (1974) are a transition from concave to flat interface at high acceleration levels, unexpected cell size and the existence of twinning and deformation. These experiments indicate that defact and twin free crystals are to be expected from growth under microgravity conditions. 
Indeed, growth of mixed InSb-GaSb crystals in Skylab showed that there were fewer twins in space-grown ingots.

\subsection{Growth of dendrites}

Under the influence of a negative temperature gradient in the liquid ahead of the solid-liquid interface, the latent heat of solidification can be conducted away by both solid and liquid phases. Such a condition promotes the growth of any projection or spike on the interface. During further growth the projection, which normally has a paraboloid shape, develops secondary and higher order spikes. The resultant growth structure is known as a dendrite. The spacings of the primary and secondary arms determine the coarseness of a solidification microstructure and influence the properties as well as compositional uniformity.

Ahead of the growing interface, both concentration and temperature gradients exist and control further growth. While the thickness of the boundary layer is normally of the same order as the radius of the tip, the effective distance of heat conduction is larger. Fredriksson (1976) concluded that convection has very little influence on the concentration fields at the very tip of the dendrite but could strongly affect the temperature fields. Taking into account the absence of convection under microgravity conditions, Fredriksson further showed that the following features are to be expected in dendrites grown under microgravity conditions :

(i) a decrease in the tendency to branch,

(ii) a decrease in primary dendrite arm spacing and its adjustment at more regular intervals,

(iii) a reduction in the remelting of dendrite arms,

(iv) increased stability in the growth direction of a dendrite and

(v) a drop in solute segregation to the interdendritic space or a decrease in microsegregation.

In addition, study of dendritic growth under microgravity conditions is expected to throw valuable light on the relative importance !of (a) anisotropy in surface energy and phase boundary reaction in controlling the crystallography of dendrite growth and (b) convection arising from density differences and solidification shrinkage in controlling macrasegregation under terrestrial conditions.

A study of the growth of dendrites in an aqueous solution of ammonium chloride was carried out on Space Processing Applications Rocket (SPAR) flight in 1975 (Johnston and Giner 1976). The results are in complete agreement with the arguments advanced by Fredriksson (1976). The dendrite arm spacing was uniform and no remelting of dendrites was noticeable. The latter observation is significant in another cantext. It is believed that the formation of equiaxed zone in the center of a casting is associated with the necking and remelting of dendrite arms (Flemings 1974). Convection has a dual role in the development of equiaxed zone as well as the chill zone on the surface of the casting. It transports the broken dendrite arms and aids in dissipating the superheat in the liquid thus enabling the transported arm to grow into a grain at its new location. When the convection is turbulent, it brings heat pulses to the interface and hastens separation of secondary arms from the dendrite. With little or no convection under conditions of 
space processing, the equiaxed zone may not obtain in a casting. An experiment on SPAR -1 at $10^{-4} \mathrm{~g}$ has, indeed, shown that no equiaxed zone resulted in a casting (Carruthers 1975).

Highly equiaxed grains can, however, arise by repeated nucleation in a melt as observed by McKannan and Poorman (1976) during electron beam melting and solidification of an Al-Cu-Mn alloy in Skylab. An electron beam was focussed on to rotating discs of the alloy of variable thickness. The beam thus traversed around a circle and depending upon the thickness of the disc either partial or complete melting across the thickness could be achieved. Identical ground-based experiments showed that the remelted region had a wide chill zone and long columnar grains. The corresponding space-processed samples on the other hand had a fine equiaxed grain structu re with a chill zane very much similar to that abtained on the ground-based experiments. The difference in microstructure in the two. cases was even more noticeable in sections that were cut through by the beam. Li (1974) interpreted the results on the basis of constitutional supercooling. According to him, the absence of convection and consequent reduction in mass transfer leads to a greater solute concentration gradient so that grain growth is retarded. Instead, independent nucleation far ahead of the interface is encouraged resulting in the fine equiaxed grain structure.

\subsection{Solidification of highly undercooled melts}

An important parameter controlling the solidification behaviour of metals and alloys is the extent to which these can be undercooled (i.e., cooled below their equilibrium freezing temperatures without inducing salidification). Conventionally, undercaoling phenomena are studied by eliminating insoluble impurities, which act as heterogeneous nucleation sites, from the melt. Towards this end the melt is either finely subdivided to increase the prabability of obtaining droplets devaid of such nucleants or the melt/droplets are conditioned by treatment with slags and other surface reactant materials (Anantharaman et al 1977; Rasmussen et al 1976). In addition, the last two decades have witnessed a remarkable growth of interest in rapid solidification of thin liquid films and tapes at the phenomenal cooling rates of $10^{6}-10^{8} \mathrm{Ksec}^{-1}$ by a variety of techniques (Jones 1976). These techniques induce a large degree of kinetic undercooling and produce many metastable solid solutions, intermetallic compounds and amorphous phases in allcys. Some of these results could be duplicated by slowly cooling droplets of liquid entrapped in solid samples. The technique called 'mushy state quenching' (Ramachandrarao et al 1979) has been developed in our labaratories based an the knowledge that such droplets undercool drastically (Southin and Chadwick 1978). In such experiments, a hypo-eutectic alloy containing about $20 \%$ by volume of the eutectic is held between the liquids and eutectic temperatures for prolonged times and repeatedly cyoled between ambient and holding temperatures. As a consequence, a considerable part of the eutectic at the grain boundaries of the sample forms globules which migrate by thermodiffusion into the interior of the pro-eutectic phase (figure 1). In each cycle the temperatures at which the droplets and residual films nucleate are monitored by thermal analysis. Invariably, the grain boundary films nucleate at a higher temperature (or lower degree of undercooling) compared to the droplets. Such a behaviour arises from the contiguity 
of grain boundary film. A single nucleation event is sufficient to solidify the films. On the contrary, the droplets behave as independent entitics in so far as nucleation is concerned and also have a lower probability of containing a heterogeneous nucleation site in them. In the experiments on aluminium-germanium alloys, the undercoolings produced have been shown to result in the formation of metastable phases identical to those abtained by rapid solidification. (Ramachandrarao et al 1979). Figure 2 shows scanning electron micrographs of the grain boundary films and droplets obtained by leaohing out matrix aluminium by electrolytic dissolution. The method offers the unique advantage of being able to monitor the temperatures at which the metastable phases nucleate. All the methods of undercooling discussed above, throw light on the important question of homogeneous nucleation temperature and the kinetic competition between stable and metastable phases. The microstructural and structural changes resulting from solidification at undercooled temperature could prone to be of technological value. For example, metallic glasses, obtained by drastically undercooling liquid alloys to a point where nucleation of the solid phases is completely suppressed, have generated immense interest by virtue of their remarkable mechanical and magnetic properties (Cahn 1980).

An important advantage of experimentation in space is the possibility of cantainerless processing of liquids. Eliminating liquid contact with the container walls automatically reduces the probability of heterogeneous nucleation and enhances the degree of undircooling. It also opens up the possibility of gaseous surfactant reactions as a means of eliminating or neutralising heterogeneous nucleation sites in the melt (Perepezko et al 1979). While no systematic undercooling experiments appear to have been performed under microgravity conditions, microstructure of aluminium-copper eutectic alloy droplets (of about $\mathbf{0 . 3}$ to $7 \mathrm{~mm}$ dia.) produced in space by passing the liquid alloy through a crack in the crucible has been discussed in terms of undercooling. Some of the globules solidified in contact with the walls of the furnace while others did so by floating freely. The former had a fine eutectic morphology and the latter exhibited a coarse distribution of the two phases. Such a structure could have resulted by drastic undercooling. However no attempt was made to measure the degree of undercooling. Calorimetric methods devised or suggested for microgravity conditions could prove to be of value in such studies (Haessner and Hemminger 1977). In this context, experiments like mushy state quenching have great utility. Ground-based experiments can be carried out on a suitably chosen alloy system and thermal analysis can be profitably employed to determine the temperatures at which the metastable phases nucleate. For reasons already discussed, space experiments can yield metastable phases by virtue of large undercooling. The ground-based experiments can be used to establish a correlation between the nature of metastable phase and the corresponding degree of undercooling.

French experiments in space propose to tackle the problcm of undercooling in silver-garmanium alloys. Malmejac (1978) has discussed the role of parameters such as cluster formation, heat of fusion, interfacial energies, etc., in controlling the expected undercooling in these alloys. In the expcriments conducted in our laboratories (Ojha and Ramachandrarao 1978), these alloys could be undercooled to an extent where the eutectic reaction was virtually suppressed. Figure 3 is a 

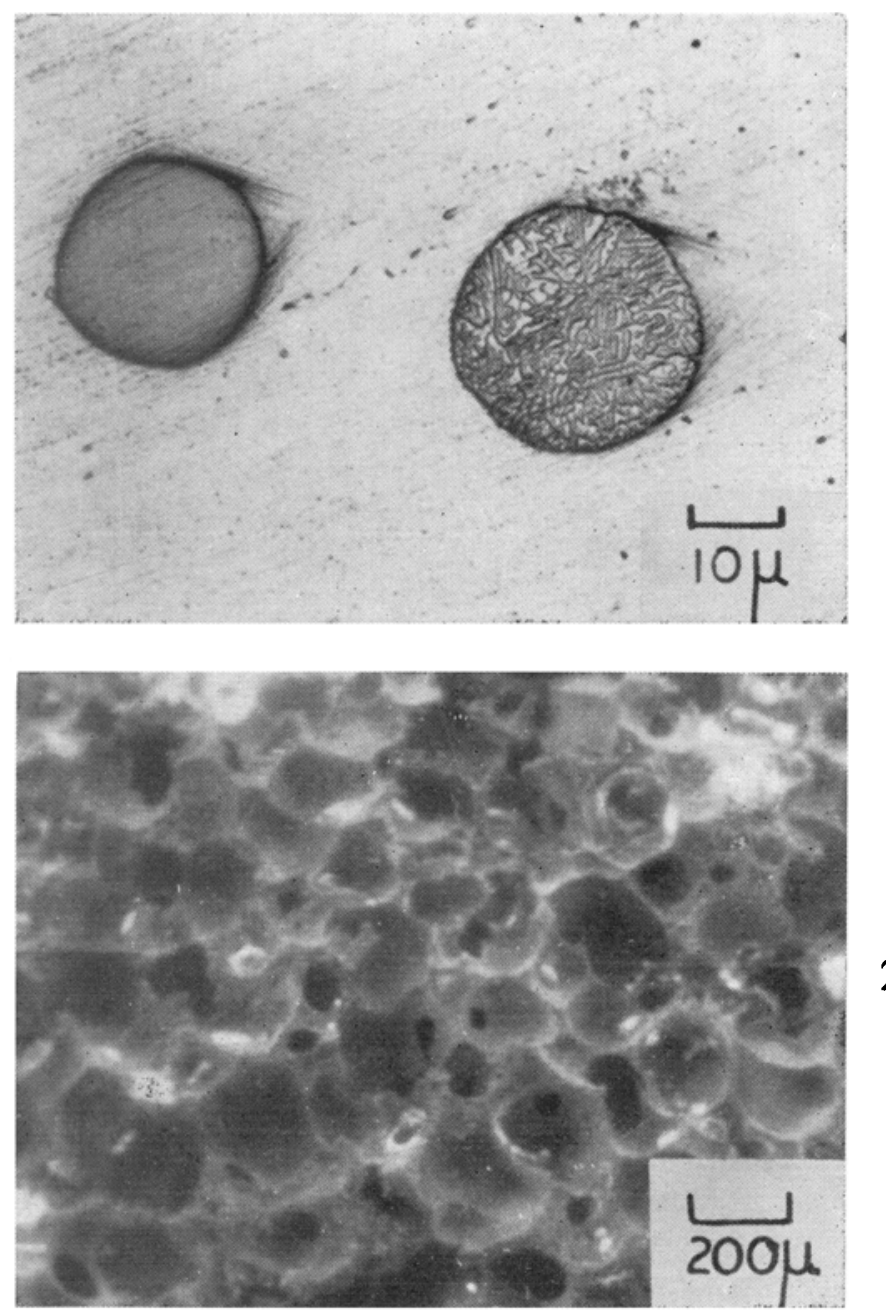

$2(a)$

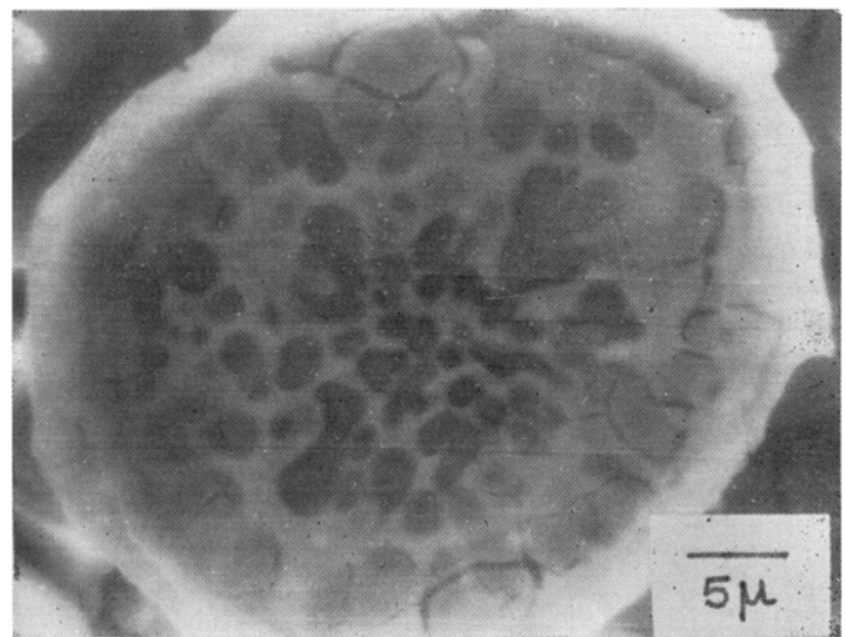

$2(b)$

Figures 1 and 2 (see Captions in page 282). 

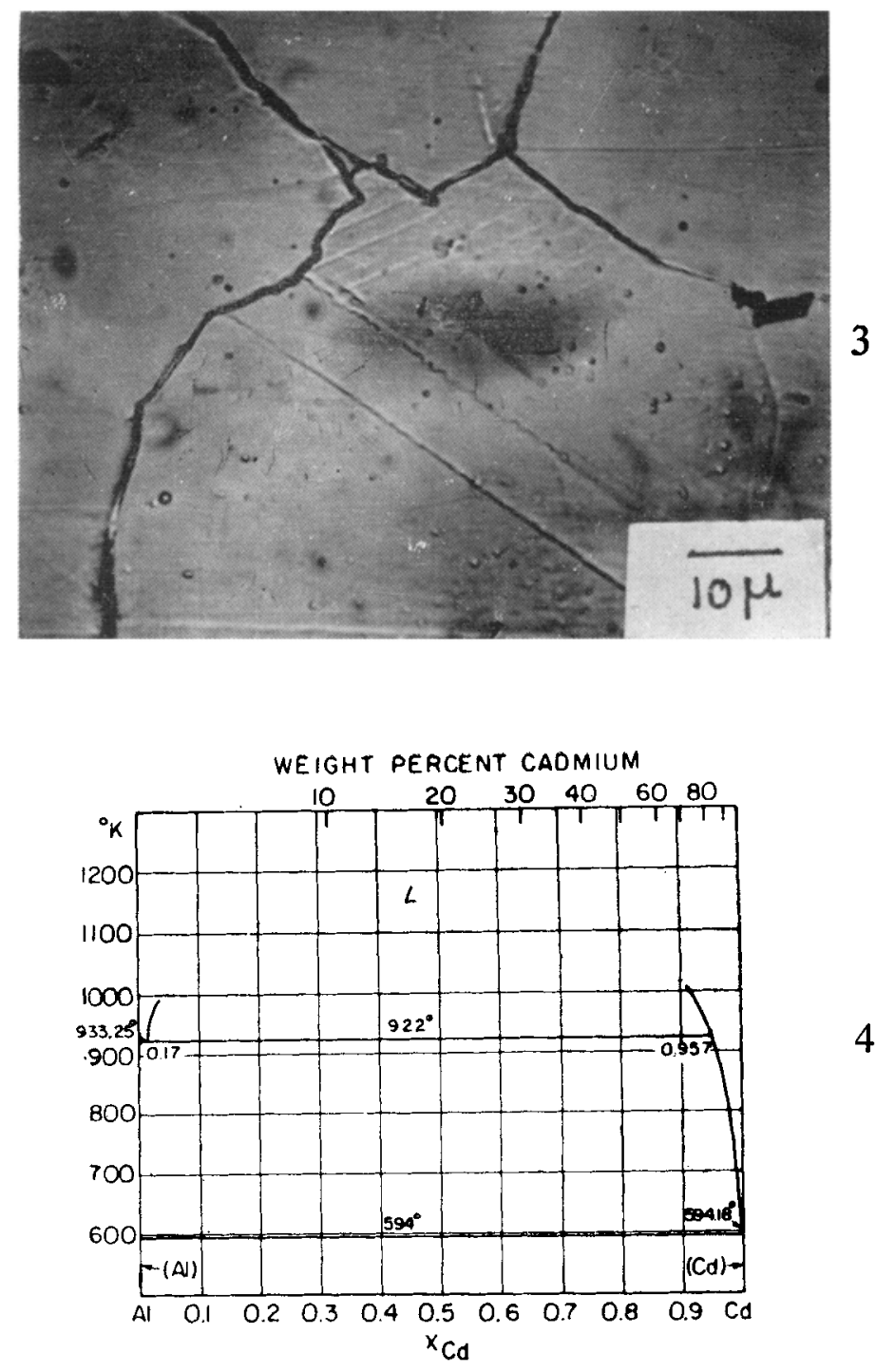

ALUMINUM - CADMIUM SYSTEM

Figure 3. Optical micrograph of an undercooled and quenched silver-14 at \% Ge alloy. The dark areas at the giain boundaries represent the eutectic phase.

Figure 4. The aluminium-cadmium monotectic system. 

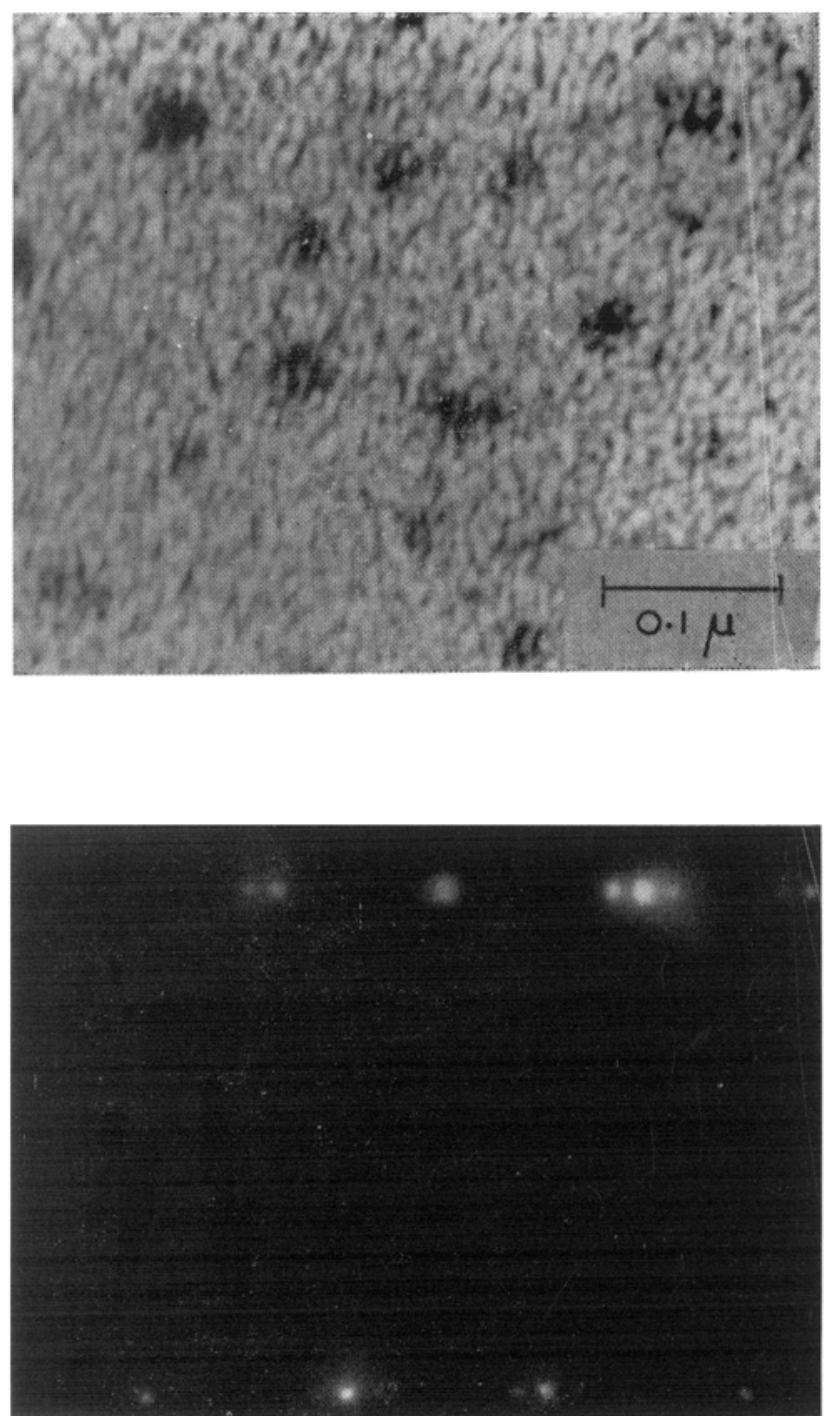

$5(b)$

Figure 5. (a) Microstructure of rapidly solidified Al-In hypomonotectic alloy. (b) Electron diffraction pattern showing satellite spots due to spinodal decomposition. 


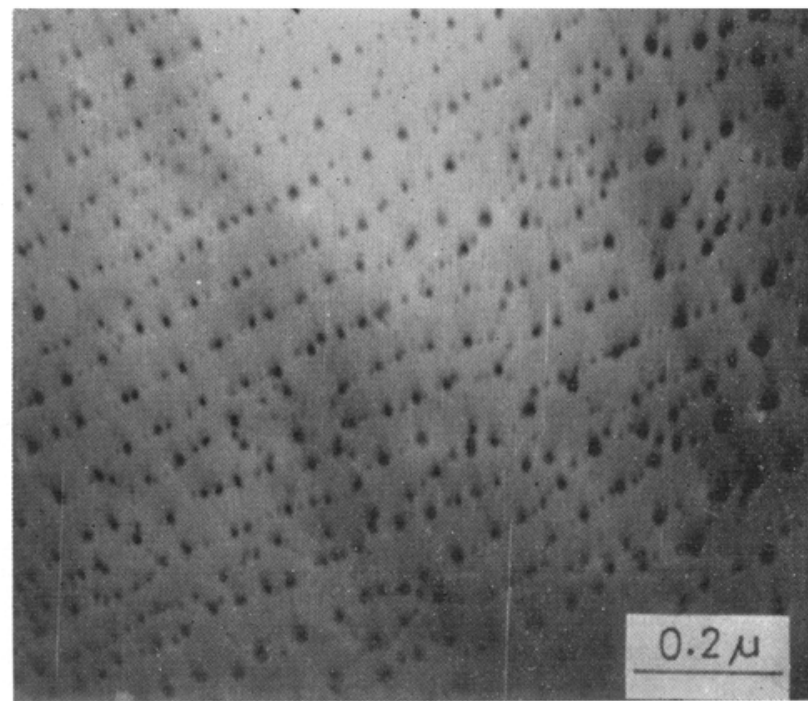

$6(a)$

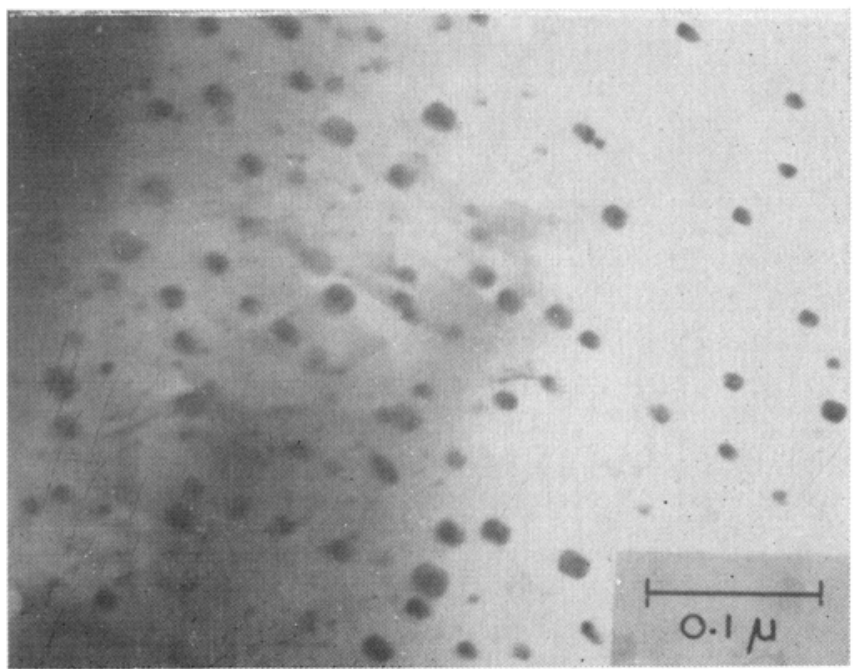

$6(b)$

Figure 6. Microstructure of a rapidly solidified monotectic Al-Cd alloy. (a) arrayed droplets. (b) random distribution of droplets. 
micrograph of a $\mathrm{Ag} 14 \% \mathrm{Ge}$ alloy. Only a small fraction of the alloy at the grain boundaries solidifies with eutectic morphology. Bulk of the alloy freeze into a metastable silver solid solution containing as much as 11 at \% $\mathrm{Ge}$ as against the maximum equilibrium concentration of 6 at $\% \mathrm{Ge}$. These experiments could serve as guidelines in analysing the results from space experiments.

\subsection{Monotectic freezing}

The monotectic reaction (figure 4 ) in which a liquid $L_{1}$ gives rise to a solid a and another liquid $L_{2}$, offers a unique opportunity of producing useful composite materials. The liquid immiscibility assaciated with the reaction can, in principle, facilitate a suitable dispersion of the second phase particles of desired dimensions. In practice, however, density differences between molten matrix and the salid phase cause sedimentation as well as convective flow which do not permit the development of the composites sought. Monotectic systems have thus attracted the attention of materials scientists as ideal candidates for composite development under microgravity conditions. Surprisingly, however, the available literature on monotectic solidification is rather limited and this has pased same problems in understanding the results from space experiments. This section briefly reviews some aspects of monotectic solidification under equilibrium, non-equilibrium and microgravity conditions.

A survey of the literature shows that the structure of manatectic alloys is sensitively dependent on solidification conditions. Process variables like growth rate, temperature gradient, thermal resistance at the heat sink-metal interface and the properties of constituent elements/phases such as density, surface tension and viscosity affect the microstructure. Even in a given monotectic system, the growth morphalogy can vary from being dendritic, composite or drop-like. The origin of such widely different microstructures in a monotectic system under steady state conditions of freezing has been qualitatively rationalized by Chadwick (1965) in terms of the interfacial energies $\gamma_{a L_{1}}, \gamma_{a L_{2}}, \gamma_{L_{1} L_{2}}$, the density difference between the phases and the rate of advance of the interface, $R$. Under conditions where $\gamma_{a L_{2}}>\gamma_{L_{1} L_{3}}+\gamma_{\alpha L_{1}}$ the droplets of $L_{2}$ cannot nucleate on $a$ and may float or sink. If they float at a rate faster than $R$, complete separation into two solid phases is possible. If $R$ is greater than the float velocity, droplets of $L_{2}$ get trapped and partially surrounded by $\alpha$. The solute atoms rejected subsequently can feed into $L_{2}$ droplets. The process as well as the resultant microstructure resemble aligned eutectic growth. It is also possible that the steady state growth may cease due to unfavourable interfacial energies, growth rates and density differences. $L_{2}$ droplets may then settle and spread over the interface as droplets of large size or continuous films. When the liquid $L_{2}$ partially wet $a$, such that

$$
\gamma_{\alpha L_{2}}=\gamma_{a L_{1}}-\gamma_{L_{1} L_{2}} \cos \theta,
$$

(where $\theta$ is the wetting angle) and other conditions are favou rable, a fibrous microstructure may result. When $\theta=0, L_{2}$ completely isolates $\alpha$ from $L_{1}$ and steadystate growth will be impossible. Only intermittent nucleation of $a$ ahead of $L_{1} L_{2}$ interface occurs leading to a bounded structure. These arguments due to Chadwick (1965) clearly bring out the role of gravitational and bu oyancy forces on the microstructure of monotectic allcys and also underline the complexity of 
monotectic solidification. The above analysis also stresses the importance of microgravity and interface growth velocity.

Recent experiments in our laboratories (Ojha and Chattopadhyay 1978; Chattopadhyay and Ramachandrarao 1980) have shown that rapid solidification of monotectio alloys, i.e., solidification at high growth velocities, can lead to a fine dispersion of initially liquid droplets of $L_{2}$, in the matrix. On further cooling, aluminium-enriched indium droplets in the $\mathrm{Al}$-In monotectic system can undercool drastically (by a mechanism similar to that in mushy state quenching) and produce a metastable face-centered cubic, indium phase (Ojha and Chattopadhyay 1978). The enhanced cooling rate can also momentarily increase the solubility of indium in the $a$ phase (aluminium). During the ensuing solid sta te cooling, the metastable solid solution decomposes spinodally. Figure 5 shows the microstructure and diftraction evidence for the spinodal decomposition. Our work on the Al-Cd monotestic system (figure 4) revealed a wide variety of microstructures (Chattopadhyay and Ramachandrarao 1980). The mode of dispersion of the cadmium-rich liquid droplets in the aluminium matrix can range from uniformly spaced arrays to a random distribution where the size of the droplets changes continu ously with change in growth conditions. In the latter case, towards the end of solidification, even continuous films of $L_{2}$ could be produced. Figure 6 shows representative microstructures. A mechanism of growth has been proposed to explain the observed microstructures (Desforges 1976).

Skylab experiments on oil-water emulsions gave the necessary impetus to the study of immiscible alloy systems (Desforges 1976). It was found that an oil-water dispersion stable for only $0.1 \mathrm{sec}$ on earth could survive without separation for over $10 \mathrm{hr}$ in space. Several experiments have been conducted in STP, SPAR and TEXUS-IT flights to study the influence of microgravity on monotectic solidification (Desforges 1976; Gelles and Markworth 1977; Alborn and Löhberg 1979; Carlberg and Fredriksson 1979). Amongst the metallic immiscible systems studied are $\mathrm{Zn}-\mathrm{Pb}, \mathrm{Zn} \rightarrow \mathrm{Bi}, \mathrm{Al} \rightarrow \mathrm{Pb}$ and $\mathrm{Al}-\mathrm{In}$. Carlberg and Fredriksson (1979) studied $\mathrm{Zn}-\mathrm{Bi}$ alloys containing 8,24 and $38 \mathrm{wt} \% \mathrm{Bi}$ in TEXUS-II. Identical experiments on earth and in space were aimed at obtaining the mean and maximum particle size distribution and the nature of the dispersed phases. Further, the alloy containing $8 \mathrm{wt} \% \mathrm{Bi}$ was subjected to two different cooling rates to assess the influence of cooling rate. Results indicated that the slower cooling rate produced larger particle size and the fraction of most particle sizes was greater. It was observed that all samples were coated with a surface film of a Bi-rich phase. The thickness of the coating was larger in case of the rapidly cooled sample. Carlberg and Fredriksson (1979) proposed that the outer layer is a result of the migration and sedimentation of the droplets. At higher Bi concentrations, the outer layer could also result from improper melting and homogenisation of the sample prior to cooling. In general, the average particle size was greater than that predicted from diffusion controlled growth which strongly points to the role of collision coalescence.

On the whole, the observation of coarse microstructures, strong separation of two liquid phases and collision coalescence were unexpected and further theoretical and experimental efforts are called for. One possible way of avoiding the problems appears to be the use of rapid solidification techniques. In this con 
nection, the experiments of Mertz et al (1975), who simulated microgravity conditions by ejecting droplets of Al-50 wt. \% in alloy and allowing them to freely fall onto a copper substrate are of significance. It was observed that relatively uniform dispersion of In-rich phase could be obtained and was considered to represent the situation under microgravity as the freezing was very rapid. High rates of cooling do not allow coalescence as was also demonstrated by our experiments. It, therefore, appears that thermodiffusion and coalescence under slow cooling rates produce the microstructure obtained in the experiments in space.

\section{Conclusions}

Experimental and theoretical attempts todate show that there is negligible convection in liquids in space and that the direct influence of gravityless enviranment is little on diffusion kineties and solidification rates. More homogeneous crystals that are defect-free can be grown in space. Dendritic growth under microgravity conditions can result in more uniformly spaced primary and sccondary arms and analysis of data from such experiments can throw light on the underlying principles of branching in dendrites. Although results from experiments on monotectic solidification ate not always consistent with expectations, it is hoped that with the introduction of rapid solidification rates some of the inconsistencies can be eliminated. Studies on undercooling of metals and alloys can help establish the role of various thermodynamic parameters of nucleation and growth and help in defining the kinetic competition between stable and metastable phases. Several techniques such as rapid solidification and mushy state quenching can be used to clarify some of the problems in undercooling of metallic melts. The knowledge acquired todate should help in the design of materials of controlled composition, microstructure and properties.

\section{Acknawledgements}

The author is indebted to Dr K Chattopadhyay and Shri Y V S S Prasad for help in the preparation of the manuscript. Critical comments and encouragement from Professor S Ranganathan and Dr S Lele are gratefully acknowledged.

\section{References}

Alborn H and Löhbcrg K 1979 AIAA Seventeenth Aerospace Sciences Meeting, New Orleans, L.A. AIAA-Paper 79--0172

Anantharaman T R, Chattopadhyay K and Ojha S N 1977 Indion Foundry J. 235

Ang C and Lacy L L 1979 Metall. Trans. 10 A 519

Cahn R W 1980 Contemp. Phys. 2143

Carlberg T and Fredriksson H 1979 Proc. Third European Sumposium on, Materials Science in Space Grenoble, 24-27 April 1979 ESA-SP-142, 233

Carruthers J R 1977 J. Cryst. Growth 42379

Chadwick G A $1965 \mathrm{Br}$. J. Appl. Phys. 161095

Chattopadhyay K and Ramachandrarao P 1980 J. Mat. Sci. 15685

Cohen M H and Turnbull D 1959 J. Chem. Phys. 311164 
Desforges C D 1976, Proc. Second European Symposium on Materials Processing in Space, Frascati, 6-8, April ESA-SP-114, 301

Flemings M C 1974 Solidification Processing (New York: McGraw-Hill)

Fredriksson H 1976, Proc. Second European Symposium on Materials Science in Space, Frascati 6-8, April ESA-SP-114, 291

Gelles S H and Markworth A J 1977 AIAA Fifteenth Aerospace Siences Meeting, New Orleans, L.A. AIAA-Paper 77-122

Haessner F and Hemminger W Hdt. Vortragsveroff Esses Werkst im Weltraum, May 1977 (391) 78

Jones $\mathrm{H}$ in Rapidly quenched metals (eds.) $\mathrm{N} \mathrm{J}$ Grant and B C Giessen, (M.L.T. Press, Cambridge), 11

Johnston M H and Baldwin D H 1974 Metall. Trans. 52395

Johnston M H and Giner C S Rept. on SPAR Experiment No. 74-21, May 1976, NASA Marshall Space Flight Centre

Li C H 1974 Report 10, contract NAS 8-29166, May 1974, Grumman Acrospace Corporation, Bethpage, N.Y.

Alborn H and Löhberg K, 1979 AIAA Seventeenth Aerospace Scionces Meeting, New Orleans, L.A. AIAA-Paper 79-0172

Malmejac Y 1978 Proc. R. Soc. (London) A361 165

Mckannan E C 1977 Aluminium 53376

McKannan E C and Poorman R M Proc. Third Space Symposium, Skylab Results Vol. 1, April 1976, NASA Marshall Space Flight Centre p. 85

Merz D, Simhan K and Winter H Report No. BMFT-FB W 75-23, Nov. 1975

Miller R I and Ruff R C 1975 J. Appl. Phys. 46208

Ojha S N and Chattopadhyay K 1978 Trans. Indian Inst. Metals 31208

Ojha S N and Ramachandrarao P 1978 Trans. Indian Inst. Metals 31295

Perepezko J H, Galup C and Rasmussen D H Proc. Third European Symp. on Materials Science in Space, Grenoble, 24-27, April 1979, ESA-SP-142, 375

Ramachandrarao P, Lal K, Singhdeo A and Chattopadhyay K 1979 Mater. Sci. Engg. 41259

Rasmussen D H, Perepezko J H and Loper C R 1976 Rapidly Quenched Metals (eds.) N J Grant and B C Giessen, (M.I.T. Press, Cambridge) 151

Reed R E, Uelhoff W and Adair H L 1977 ORNL Report 76-113 1976, see also McKannan E, Aluminium $\mathbf{5 3} 376$

Southin R T and Chadwick G A 1978 Acta Metall. 26223

SPAR Experiment No. 74-21 (see Carruthers 1977)

Stark J P 1978 Acta Metall. 261139

Turnbull D 1961 Trans. Metall. Soc. AIME 221422

Ukanwa A, Skylab results Third Space Processing Symposium (ed.) M P L Siebel 1 and 2 (NASA) June 1974

Yue J T and Voltmer F W $1975 \mathrm{~J}$. Cryst. Growth 29329

Figure 1. Droplets in a aluminium-germanium hypo-eutectic alloy formed by holding in the solid + liquid region. The featureless drop contains the metastable phases.

Figure 2. Scanning electron micrographs of undercooled and rapidly solidified (a) liquid films and (b) a droplet in an aluminium-germanium hypo-eutectic alloy. The samples were produced by electrolytic extraction of the aluminium matrix. 